\title{
On Liouvillian Solutions of Third Order Homogeneous Linear Differential Equations
}

\author{
Noura Okko \\ Correspondence: Noura Okko, Prof. Dep. of Math. Faculty of Science, Tishreen University, Lattakia, Syria
}

Received: March 7, 2019 Accepted: May 5, 2019 Online Published: May 8, 2019

doi:10.5539/jmr.v11n3p14 URL: https://doi.org/10.5539/jmr.v11n3p14

\begin{abstract}
In this article we will consider third order homogeneous differential equations: $L(y)=y^{\prime \prime \prime}+a_{1} y^{\prime}+a_{0} y \quad\left(a_{0}, a_{1} \in k\right)$ whose Galois group $\mathfrak{S}(L)$ is imprimitive. This case is characterised by the fact that the third symmetric power equation $L^{\circledR 3}(y)=0$ has an exponential solution whose square is rational (Singer \& Ulmer 1993). If $L(y)=0$ has a Liouvillian solution $z$ whose logarithmic derivative $u=z^{\prime} / z$ is algebraic over a differential field $\left(k,{ }^{\prime}\right)$, we will give an algorithm to find the relation between $a_{0}, a_{1}$, the semi-invariant $S=Y_{1} Y_{2} Y_{3}$ which is unique up to multiplication by a constant, the coefficients $c_{0}, c_{1}$ of the minimal polynomial $P(U)$ of $u$ and their derivatives. The aim of this work is to diminutize the number of constants $C_{m}$ stated in the algorithm of Singer \& Ulmer (Singer \& Ulmer 1993 Algorithm p. 31) whose determination is not easy to do, and we will achieve this by using Groebner Basis.
\end{abstract}

Keywords: differential Galois Theory, Liouvillian solutions, Groebner Basis, invariant theory, representation theory

\section{Introduction}

This section is a recall of some definitions in Differential Galois theory that we will depend on in our study. In the second section we will give an algorithm to find the relation between $a_{0}, a_{1}$, the semi-invariant $S=Y_{1} Y_{2} Y_{3}$ which is unique up to multiplication by a constant, the coefficients $c_{0}, c_{1}$ of the minimal polynomial $P(U)$ of $u$ and their derivatives. The aim of this work is to diminutize the number of constants $C_{m}$ stated in the algorithm of Singer \& Ulmer (Singer \& Ulmer 1993 Algorithm p. 31) whose determination is not easy to do, and we will achieve this by using Groebner Basis, and in the third section we will expose the codes Maple and Magma we have used for more knowledge about Invariant Theory refer to (Benson 1993, Cox 1992, Sturmfels 1993)

\section{Differential Galois theory}

Definition 1. (Singer \& Ulmer 1998 p.3) A differential field $\left(k,{ }^{\prime}\right)$ is a field $k$ together with a derivation' in $k$. The set of all constants $C=\left\{a \in k, a^{\prime}=0\right\}$ is a subfield of $\left(k,{ }^{\prime}\right)$.

Definition 2. (Singer \& Ulmer 1998 Defination1.1.) A differential field extension $(K, \Delta)$ of $(k, \delta)$ is a Liouvillian extension if there is a tower of fields :

$$
k=K_{0} \subset K_{1} \subset \cdots \subset K_{m}=K
$$

Where $K_{i+1}$ is a simple field extension $K_{i}\left(\eta_{i}\right)$ of $K_{i}$, such that one of the following holds:

$>\eta_{i}$ is algebraic over $K_{i}$,

$>\delta\left(\eta_{i}\right)=f_{i} \in K_{i}$ (extension by an integral $\left.\eta_{i}=\int f_{i}\right)$,

$>\delta\left(\eta_{i}\right) / \eta_{i}=f_{i} \in K_{i}$ (extension by the exponential of an integral $\eta_{i}=e^{\int f_{i}}$ ).

A function contained in a Liouvillian extension of $k$ is called a Liouvillian function over $k$.

Definition 3. (Fakler 1997 p. 29) Let $\left(k,,^{\prime}\right)$ be a differential field of characteristic 0 and $C$ is its field of constants algebraically closed. Consider the following ordinary homogeneous linear differential equation:

$$
L(y)=y^{(n)}+a_{n-1} y^{(n-1)}+\cdots+a_{1} y^{\prime}+a_{0} y=0 \quad\left(a_{i} \in k\right)
$$

over $\left(k,{ }^{\prime}\right)$ with a system $\left\{y_{1}, \ldots, y_{n}\right\}$ of fundamental solutions. By extending the derivation' to a system of fundamental solutions and by adjunction of these solutions and their derivatives to $\left(k,{ }^{\prime}\right)$ in a way the field of constants does not change, one gets $K=k\left\langle y_{1}, \ldots, y_{n}\right\rangle$, the so-called Picard-Vessiot extension (PVE) of $L(y)=0$. With these assumptions, the PVE of $L(y)=0$ always exists and is unique up to differential isomorphism. This extension plays the 
same role for a differential equation as a splitting field for a polynomial equation.

Definition 4. (Fakler 1997 p. 29) The set of all automorphisms of $K$, which fix $\left(k,,^{\prime}\right)$ elementwise and commute with the derivation in $K$ is a group, it is the differential Galois group $\mathfrak{S}(K / k)=\mathfrak{S}(L)$ of $L(y)=0$.

Let $L(y)=y^{(n)}+a_{n-1} y^{(n-1)}+\cdots+a_{1} y^{\prime}+a_{0} y=0\left(a_{i} \in k\right)$ be an $n-t h$ order linear diferential equation with Galois group $\mathfrak{S}(L) \in G L(n, C)$. To a basis $\left\{y_{1}, \ldots, y_{n}\right\}$ of the solution space of $L(y)=0$ we associate the evaluation morphism:

$$
\begin{aligned}
\Phi:\left[Y_{1}, \ldots, Y_{n}\right] & \rightarrow K \\
Y_{i} & \mapsto y_{i}
\end{aligned}
$$

The value of a semi-invariant of $\mathfrak{S}(L) \in G L(n, C)$ is its image in $K$ under the above evaluation morphism.

The morphism $\Phi$ has the following properties:

a. $\Phi$ restricts to a bijection between linear forms and solutions of $L(y)=0$

b. Semi-invariants of finite order $n$ are sent to the $n-t h$ roots of elements of $k$. In Particular invariants are fixed under the action of $\mathfrak{S}(L)$ and are sent to elements of $k$.

Definition 5. (Singer \& Ulmer 1997 Definition 1) Let $L(y)=0$ be a homogeneous linear diferential equation of order $n$ and let $\left\{y_{1}, \ldots, y_{n}\right\}$ be a fundamental system of solutions. The differential equation $L^{\circledR m}(y)=0$ whose solution space, denoted $S_{m}$ is spanned by monomials of degree $m$ in $y_{1}, \ldots, \mathrm{y}_{n}$ is called the $m-$ th symmetric power of $L(y)=0$.

An algorithm to construct $L^{(\circledast m}(y)=0$ is given in (Singer 1980). The value of a semi-invariant of degree $m$ and order $j$ is an $j-$ th root of a solution of $L^{\circledR m}(y)=0$ (Singer \& Ulmer 1993 Lemma 1.6.).

Definition 6. (Singer \& Ulmer 1997 p.2) Let $L(y)=y^{(n)}+a_{n-2} y^{(n-2)}+\cdots+a_{1} y^{\prime}+a_{0} y=0\left(a_{i} \in k\right)$ be a linear differential equation of order $n$ over $k$. A solution of (1) in $k$ is a rational solution, a solution in an algebraic extension of $k$ is an algebraic solution, , a solution whose logarithmic derivative is in $k$ is an exponential solution, a solution belonging to a field obtained from $k$ by successive adjunction of exponentials, integrals and algebraic functions is a Liouvillian solution.

Definition 7. ( Ulmer 1994. P.3) Let $G \subset G L(V)$ be a linear group acting irreducibly on the vector space $V$ of dimension $n$ over $C$. Then $G$ is said to be imprimitive if there exist subspaces $\left\{V_{1}, \ldots, V_{d}\right\}$ with $d \succ 1$ such that $V=V_{1} \oplus \ldots \oplus V_{d}$ and, for each $\sigma \in G$, the mapping $V_{i} \rightarrow \sigma\left(V_{i}\right)$ is a permutation of the $\operatorname{set}^{A}=\left\{V_{1}, \ldots, V_{d}\right\}$ The set $A$ is called a system of imprimitivity of $G$. If all the subspaces $V_{i}$ are of one dimension, then $G$ is called monomial. An irreducible group $G \subseteq G L(V)$ which is not imprimitive is called primitive.

for more knowledge about Differential Galois theory refer to Magid 1994, Kolchin 1948.

\section{Algorithm to Diminutize the Number of Constants $C_{m}$ Stated in the Algorithm of Singer \& Ulmer 1993}

In this section we will consider linear ordinary differential equations of third order the form: $L_{S L}(y)=y^{\prime \prime \prime}+a_{1} y^{\prime}+a_{0} y \quad\left(a_{0}, a_{1} \in k\right)$ without losing the Liouvillian character of the solutions (Kolchin 1948, p.184, OKKO 2018 Remark. P. 51), whose Galois group $\mathfrak{S}(L)$ is imprimitive. This case is characterised by the fact that the third symmetric power $L^{\circledR 3}(y)=0$ has an exponential solution whose square is rational or equivalently it has a semi-invariant of degree 3 and of order $\leq 2$ exists (cf. Singer \& Ulmer 1993 Theorem 4.6). If $L(y)=0$ has a Liouvillian solution $Z$ whose logarithmic derivative $u=z^{\prime} / z$ is algebraic over $a$ differential field $(k$,$) , we will$ give an algorithm to find the coefficients of the minimal polynomial $P(U)$ of $u$ in terms of $a_{0}, a_{1}$, the semi-invariant $S=Y_{1} Y_{2} Y_{3}$ which is unique up to multiplication by a constant, and their derivatives by using Groebner Basis. Befor this we will introduce a lemma (Ulmer 2003, Lemma 3.1). ) and recall it.

Let $R_{m}=k\left[Z_{1,0}, \ldots, Z_{m, n-1}\right]$ be the polynomial ring in $n m$ variables. We will take the same notations of Felix Ulmer in

(Ulmer 2003): $W_{i_{1}, \ldots, i_{m}}$ denotes for the monic polynomial corresponding to $\sum_{\sigma \in S_{m}} Z_{\sigma(1)}^{\left(i_{1}\right)} Z_{\sigma(2)}^{\left(i_{2}\right)} \cdots Z_{\sigma(m)}^{\left(i_{m}\right)} \in R_{m}$

for integers $n \succ i_{1} \geq i_{2} \geq \cdots \geq i_{m} \geq 0$. Let $S=Z_{1} Z_{2} \cdots Z_{m} \in R_{m}$ and $S^{\prime}, \ldots, S^{(j)}$ the first $j$ derivatives of

$S$, then $S$, its derivatives and the $N=\left(\begin{array}{c}m+n-1 \\ n-1\end{array}\right)$ polynomials $W_{i_{1}, \ldots, i_{m}}\left(\in R_{m}\right)$ are related by a matrix 


$$
A_{j} \in(k,(j+1) \times N):
$$

$$
\left(\begin{array}{c}
S \\
S^{\prime} \\
\ldots \\
S^{(j)}
\end{array}\right)=A_{j}\left(\begin{array}{c}
W_{0, \ldots .0} \\
W_{1,0, \ldots .0} \\
\ldots \\
W_{n-1, \ldots, n-1}
\end{array}\right)
$$

The theorem 3 and its proof in (Singer \& Ulmer 1997.) show that if $g \in K$ is an exponential solution of $L^{(\subseteq m}(y)=0$ and $g=\Psi_{z_{1}, \ldots, z_{m}}(S)$, for $m \in \mathbb{N}$ and $z_{1}, \ldots, z_{m}$ are $m$ solutions ,different from zero, of the equation $L_{S L}(y)=0$.

Then for all polynomial $P(U) \in K[U]$ :

$$
\begin{aligned}
& P(U)=\left(\prod_{i=1}^{m} z_{i}\right) U^{m}-\left(z_{1}^{\prime} z_{2} \ldots z_{m}+\ldots+z_{1} z_{2} \ldots z_{m}^{\prime}\right) U^{m-1}+\ldots+(-1)^{m} \prod_{i=1}^{m} z_{i}^{\prime} \\
& =\Psi_{z_{1}, \ldots, z_{m}}\left(W_{0, \ldots, 0}\right) U^{m}-\Psi_{z_{1}, \ldots, z_{m}}\left(W_{1,0, \ldots, 0}\right) U^{m-1}+\cdots+(-1)^{m} \Psi_{z_{1}, \ldots, z_{m}}\left(W_{1, \ldots, 1}\right)
\end{aligned}
$$

the polynomial :

$$
Q(U)=\frac{P(U)}{\prod_{i=1}^{m} z_{i}}
$$

is in the ring $K[U]$. And in this case all roots $u_{i}$ of $Q(U)$ give Liouvillian solutions $e^{\int u_{i}}$ of $L(y)=y^{\prime \prime \prime}+a_{2} y^{\prime \prime}+a_{1} y^{\prime}+a_{0} y \quad\left(a_{0}, a_{1}, a_{2} \in k\right)$ ( cf. Remark. OKKO 2018).

Lemma 3.1. (Ulmer F. 2003, Lemma 3:1). For a third order linear differential equation $L(y)=y^{\prime \prime \prime}+a_{1} y^{\prime}+a_{0} y$ over

$k$ the order of $L^{(5)}(y)=0$ is 7,9 or 10 . The order of $L^{(53}(y)=0$ is 7 if and only if $2 a_{0}=a_{1}^{\prime}$. In this case

$$
\left.L(\mathrm{y})=\tilde{L}^{(} 2\right)(y) \text { where } \tilde{L}(\mathrm{y})=y^{\prime \prime}+\frac{a_{1}}{4} y .
$$

Proof: Let $\Phi_{m}$ be the $\mathfrak{S}$-morphism obtained by restriction of the evaluation morphism $\Phi$ to the vector space of homogeneous forms of degree $m$ of $C\left[Y_{1}, Y_{2}, Y_{3}\right]$ (Singer \& Ulmer 1993 Lemma 3.5). Because $\Phi_{1}$ is a bijection, (Lemma 3.5 (4) in Singer \& Ulmer 1993) implies that the kernel of $\Phi_{2}$ is at most of dimension 1, i.e. $L^{\otimes_{2}}(y)=0$ is of order at least 5. If $\Phi_{2}$ is a bijection, then (Lemma 3.5 (4) in Singer \& Ulmer 1993) implies that $L^{\circledR 3}(y)=0$ is of order 9 or 10. Suppose now that the kernel of $\Phi_{2}$ is of dimension 1 and generated by $F_{2} \in C\left[Y_{1}, Y_{2}, Y_{3}\right]$. Note that $F_{2}$ must be irreducible since otherwise $\Phi_{1}$ is not a bijection. The homogeneous polynomials of degree 3 divisible by $F_{2}$ correspond to the $C$-Span of $Y_{1} F_{2}, Y_{2} F_{2}$ and $Y_{3} F_{2}$ are in the kernel of $\Phi_{3}$, showing $L^{\circledR 3}(y)=0$ is of order $\leq 7$. If $L^{\Theta_{3}}(y)=0$ is of order $\leq 6$, it must exist a polynomial $F_{3} \in C\left[Y_{1}, Y_{2}, Y_{3}\right]$ in the kernel of $\Phi_{3}$ which is not a multiple of $F_{2}$. If $F_{2}$ or $F_{3}$ is of degree zero in $Y_{3}$, then we get a non constant homogeneous polynomial $F \in C\left[Y_{1}, Y_{2}\right]$ whose evaluation via $\Phi$ is zero. Since $F$ is homogeneous and $C$ is algebraically closed, $F$ factors as a product of linear forms $\prod_{i}\left(\alpha_{i} Y_{1}-\beta_{i} Y_{2}\right)$.The evaluation via $\Phi$ gives a linear relation between the basis elements $y_{1}, y_{2}$ and thus a contradiction. Denote $F_{2,3} \in C\left[Y_{1}, Y_{2}\right]$ the resultant of $F_{2}$ and $F_{3}$ with respect to $Y_{3}$. Since $F_{2}$ and $F_{3}$ are relatively prime and are both of degree $\geq 1$ in $Y_{3}$, we get that $F_{2,3} \in C\left[Y_{1}, Y_{2}\right]$ is homogeneous of degree $\geq 1$. Arguing as above with $F$, we get a contradiction. Thus $L^{\circledR 3}(y)=0$ is of order 7 in this case. This proves the first assertion. We want to characterise the fact That $L^{(3)}(y)=0$ is of order 7 or equivalently that $L^{(92}(y)=0$ is of order 5 . This has been done(Singer 1985) Lemma 3.4, but we will give a proof using the introduced formalism. According to section 2.1 we have that $L^{\complement 2}(y)=0$ is of order $\leq 5$ if and only if the matrix $A_{5}$ is not invertible (i.e.if and only if $\left(z_{1} z_{2} z_{3}\right), \ldots,\left(z_{1} z_{2} z_{3}\right)^{5}$ are linearly dependent). Performing a fraction free Gaussian elimination on the matrix $\left(A_{5}\right)^{t}$ we get: 


$$
\left[\begin{array}{cccccc}
1 & 0 & 0 & -2 a_{0} & -2 a_{0}^{\prime} & 2 a_{1} a_{0}-2 a_{0}^{\prime \prime} \\
0 & 1 & 0 & -a_{1} & -5 a_{0}-a_{1}^{\prime} & -7 a_{0}^{\prime}+a_{1}^{2}-a_{1}^{\prime \prime} \\
0 & 0 & 2 & 0 & -8 a_{1} & -10 a_{1}^{\prime}-10 a_{0} \\
0 & 0 & 0 & 6 & 0 & -30 a_{1} \\
0 & 0 & 0 & 0 & 36 & 0 \\
0 & 0 & 0 & 0 & 0 & 108\left(a_{1}^{\prime}-2 a_{0}\right)
\end{array}\right]
$$

Therefore $L^{\circledR 3}(y)=0$ is of order 7 if and only if $a_{1}^{\prime}=2 a_{0}$.A direct computation shows that in this case $L(y)=\tilde{L}^{\Im 2}(y)$.

Now we will give an algorithm to find the coefficient of $P(U)$ in terms of $a_{0}, a_{1}$, the semi-invariant $S=Y_{1} Y_{2} Y_{3}$ and their derivatives :

\section{The Algorithm:}

$>$ Write $S$ and its derivatives in terms of the polynomials $w_{i, j, k}$, the coefficients $a_{1}, a_{0}$ and their derivatives (see section 3. , NormalForm1 and NormalForm2 procedures).

Calculate Groebner Basis $G R$ of the ideal $I=\left\langle S-w_{0,0,0}, \ldots, S^{(9)}-\ldots, c_{0}-w_{1,1,1}, c_{1}-w_{1,1,0}\right\rangle$

$$
\begin{aligned}
& \subset R=k\left\langle w_{0,0,0}, w_{1,0,0}, w_{2,0,0}, w_{1,1,0}, w_{2,1,0}, w_{1,1,1}, w_{2,2,0}, w_{2,1,1}, w_{2,2,1}, w_{2,2,2}, a_{0,0}\right. \\
& \left.a_{1,0}, a_{0,1}, \ldots, a_{0,6}, a_{1,6}, S, S^{\prime}, S^{\prime \prime}, \ldots, S^{(9)}, c_{0}, c_{1}\right\rangle
\end{aligned}
$$

such that the order on the ring $R$ is the elimination order on the first 10 variables, so we can find relations between $s, a_{1}, a_{0}$, their derivatives and the coefficients $c_{1}=z_{1}^{\prime} z_{2}^{\prime} z_{3}+z_{1}^{\prime} z_{2} z_{3}^{\prime}+z_{1} z_{2}^{\prime} z_{3}^{\prime}$,

$c_{0}=-z_{1}^{\prime} z_{2}^{\prime} z_{3}^{\prime}$ of $P(\mathrm{U})$ in the ring $k\left[S, S^{\prime}, S^{\prime \prime}, \ldots, S^{(9)}\right]$ (see section 3., $s, a_{1}, a_{0}$ and their derivatives procedure). After calculating Groebner Basis $G R$ we find that its latest six elements have only the variables $s, a_{0}, a_{1}$, their derivatives and the coefficients $c_{0}, c_{1}$.

$>$ Eliminate one more time the coefficient $c_{0}$ considred as a variable in the ring $k\left[c_{0}, c_{1}, a_{0}, a_{1}, a_{0}^{\prime}, a_{1}^{\prime}, \ldots, a_{0}^{(6)}\right.$, $\left.a_{1}^{(6)}, s, s^{\prime}, s^{\prime \prime}, \ldots, s^{(9)}\right]$ by calculating Groebner Basis $G r$ of the ideal $i d=\left\langle G R_{16}, \ldots, G R_{21}\right\rangle$

(see section 3 , eliminate $c_{0}$ ). So we obtain a relation between the variables $S, S^{\prime}, \ldots, S^{(9)}, a_{0}, a_{0}^{\prime}, \ldots, a_{0}^{(6)}$, $a_{1}, a_{1}^{\prime}, \ldots, a_{1}^{(6)}$ and $c_{1}$.

We determin the value of $c_{1}$ with Maple. We substitute this value in the element of Groebner Basis

$G r$ which contains $S, a_{1}, a_{0}$, their derivatives and the coefficients $c_{0}, c_{1}$ in order to express $c_{0}$ in terms of $S, a_{1}, a_{0}$ and their derivatives. In reality, the element $G r$ we speak about is $G r_{4}$, whatever the order of the symmetric equation $L^{\circledR 3}(\mathrm{y})=0$ is 7,9 or 10 .

$$
\begin{aligned}
& G r_{4}=c_{0} a_{0}^{2} a_{1}-\frac{7}{9} c_{0} a_{0}^{\prime 2}+\frac{2}{3} c_{0} a_{0} a_{0}^{\prime \prime}+\frac{43}{20} a_{0}^{3} a_{1} S+\frac{1}{4} a_{0} a_{1}^{2} a_{0}^{\prime} S-\frac{1}{30} a_{0}^{2} a_{1}^{2} a_{0}^{\prime} S^{\prime}+\frac{1}{3} a_{1}^{3} a_{0}^{\prime} S^{\prime} \\
& -\frac{1}{4} a_{0} a_{1}^{3} S^{\prime \prime}+\frac{63}{20} c_{1} a_{0}^{3}+\frac{1}{4} c_{1} a_{0} a_{1} a_{0}^{\prime}-\frac{23}{144} a_{0} a_{0}^{\prime 2} S+\frac{11}{240} a_{0}^{2} a_{0}^{\prime \prime} S-\frac{13}{48} a_{0} a_{1} a_{0}^{\prime \prime \prime} S-\frac{17}{80} \\
& a_{0}^{2} a_{0}^{\prime} S^{\prime}-\frac{67}{54} a_{1} a_{0}^{\prime 2} S+\frac{169}{144} a_{0} a_{1} a_{0}^{\prime \prime} S^{\prime}-\frac{111}{80} a_{0}^{3} S^{\prime \prime}+\frac{5}{12} a_{0} a_{1} a_{0}^{\prime} S^{\prime}+\frac{17}{24} a_{0}^{2} a_{1} S^{\prime \prime \prime} \\
& -\frac{49}{108} a_{1}^{2} a_{0}^{\prime} S^{\prime \prime \prime}+-\frac{49}{144} a_{0} a_{1}^{2} S^{(4)}-\frac{1}{9} c_{1} a_{0}^{\prime} a_{0}^{\prime \prime}+\frac{1}{12} c_{1} a_{0} a_{0}^{\prime \prime \prime}+\frac{1}{36} a_{0}^{\prime} a_{0}^{(4)} S-\frac{1}{48} a_{0} a_{0}^{(5)} S \\
& +\frac{1}{6} a_{0}^{\prime} a_{0}^{\prime \prime \prime} S^{\prime}-\frac{49}{108} a_{1}^{2} a_{0}^{\prime} S^{\prime \prime \prime}+-\frac{49}{144} a_{0} a_{1}^{2} S^{(4)}-\frac{1}{9} c_{1} a_{0}^{\prime} a_{0}^{\prime \prime} \\
& -\frac{7}{48} a_{0} a_{0}^{(4)} S^{\prime}+\frac{4}{9} a_{0}^{\prime} a_{0}^{\prime \prime} S^{\prime \prime}-\frac{11}{24} a_{0} a_{0}^{\prime \prime \prime} S^{\prime \prime}+\frac{14}{27} a_{0}^{\prime \prime \prime 2} S^{\prime \prime \prime}
\end{aligned}
$$

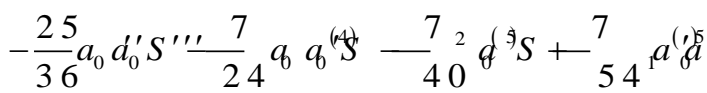




$$
-\frac{7}{72} a_{0} a_{1} S^{(6)}-\frac{1}{108} a_{0}^{\prime} S^{(7)}+\frac{1}{144} a_{0} S^{(8)}
$$

Examples.

$>L^{(3)}(y)=0$ is of order 7 .

Example1. Take the equation :

$$
L(y)=\frac{d^{3} y}{d x^{3}}+\frac{32 x^{2}-27 x+27}{36 x^{2}(x-1)^{2}} \frac{d y}{d x}-\frac{64 x^{3}-81 x^{2}+135 x-54}{72 x^{3}(x-1)^{3}} y
$$

whose symmetric power $L^{(S 3}(y)=0$ is of order 7 . The $\mathbb{Q}$ - vector space of Liouvillian solutions of $L^{(5)}(y)=0$ is generated by only one solution $s=x^{4}-2 x^{3}+x^{2}$. By using our algorithm, we obtain the coefficients of the minimal polynomial $P(U)$ :

$c_{3}=s=x^{4}-2 x^{3}+x^{2}$

$c_{2}=s^{\prime}=4 x^{3}-6 x^{2}+2 x$

$c_{1}=25313961574400 x^{11}-122936959713280 x^{10}+369414924755712 x^{9}$

$-759762796466484 x^{8}+1160223530219379 x^{7}-1352965374477072 x^{6}$

$+1190804183867628 x^{5}-779912777349666 x^{4}+353889936963693 x^{3}$

$-95718851436546 x^{2}+11393791055748 x-\frac{41822280936}{108}\left(21010579456 x^{9}\right.$

$-79774543872 x^{8}+234889910208 x^{7}-435358558917 x^{6}+634598874729 x^{5}$

$-673422918633 x^{4}+534201757263 x^{3}-298530368262 x^{2}+96095161620 x$

$-12699314136)$

$c_{0}=40936268627968 x^{12}-221509596151808 x^{11}+669402865431552 x^{10}$

$-1391212752866640 x^{9}+2084599015102737 x^{8}-2335386769620696 x^{7}$

$+1894909901929902 x^{6}-1033536296671596 x^{5}+272124855869121 x^{4}$

$+84555330577452 x^{3}-101906800574184 x^{2}+33293137560192 x$

$-\frac{3816809262000}{216} x(x-1)\left(64 x^{3}-81 x^{2}+135 x-54\right)\left(328290304 x^{6}\right.$

$-830984832 x^{5}+1925952309 x^{4}-2335090518 x^{3}+2196559377 x^{2}$

$-1191608820 x+235172484)$

$>\quad L^{(5)}(y)=0$ is of order 9 .

Example2. Take the equation:

$$
\begin{aligned}
L(y)= & \frac{d^{3} y}{d x^{3}}+\frac{8 x-3}{x(x-1)} \frac{d^{2} y}{d x^{2}}+\frac{3264 x^{2}-2452 x+729}{108 x^{2}(x-1)^{2}} \frac{d y}{d x} \\
& -\frac{71280 x^{3}-80410 x^{2}+47466 x-10935}{1458 x^{3}(x-1)^{3}} y
\end{aligned}
$$

(example 4.5 in Ulmer 2003.) that can be transferred to the equation:

$$
L_{S L}(y)=\frac{d^{3} y}{d x^{3}}+\frac{96 x^{2}-76 x+81}{108 x^{2}(x-1)^{2}} \frac{d y}{d x}-\frac{2592 x^{3}-3068 x^{2}+5400 x-2187}{2916 x^{3}(x-1)^{3}} y
$$

by using procedure transfereL with Maple program (Okko 2018). The equation $L_{S L}(y)$ is irreducible, its differential Galois group is $G_{162}$ and its third symmetric power $L^{(3)}(y)=0$ is of order 9 . The value of the semi-invariant is $s=\sqrt{(x-1)^{5}} \sqrt{x^{3}}$ whose square is rational and it generates the vector space of Liouvillian solutins of $L^{\circledR 3}(y)=0$. By 
using our algorithm we obtain the coefficients of the minimal polynomial $P(U)$ :

$$
\begin{aligned}
& c_{3}=s=\sqrt{(x-1)^{5}} \sqrt{x^{3}} \\
& c_{2}=s^{\prime}=\frac{5}{2} \sqrt{x^{3}} \sqrt{(x-1)^{3}}+\frac{3}{2} \sqrt{x} \sqrt{(x-1)^{5}} \\
& c_{1}=\frac{1}{432}\left(27680816981606400 x^{12}-146369305291456512 x^{11}+460721269652889600 x^{10}\right. \\
& -1009013808719533056 x^{9}+1645470628884383488 x^{8}-2078192403916191552 x^{7} \\
& +2032861092998975552 x^{6}-1522715003967278160 x^{5}+839232768599394624 x^{4} \\
& -307532640935458044 x^{3}+62289431318500884 x^{2}-4200887273556699 x \\
& -231958332276525) / \sqrt{x} \sqrt{x-1}\left(5743767158784 x^{9}-20393922244608 x^{8}\right. \\
& +60302794885632 x^{7}-109841060253248 x^{6}+161225407720576 x^{5} \\
& \left.-171833666743152 x^{4}+138087079432992 x^{3}-79030988627868 x^{2}+25962912325800 x-3471675001929\right) \\
& c_{0}=\frac{1}{23328}\left(1208622863106441216 x^{12}-5620819173370232832 x^{11}+15699354875538112512 x^{10}\right. \\
& -30138292242681495552 x^{9}+41327897457410536960 x^{8}-42973740430560205568 x^{7} \\
& +32763357563840003520 x^{6}-18873014663604337920 x^{5}+8966085528248791344 x^{4} \\
& -3863141918254277136 x^{3}+1563648342203575476 x^{2}-500362208768814480 x \\
& +79072079758122051) / \sqrt{x^{3}} \sqrt{x-1}\left(5743767158784 x^{9}-20393922244608 x^{8}\right. \\
& +60302794885632 x^{7}-109841060253248 x^{6} \\
& +161225407720576 x^{5}-171833666743152 x^{4}+138087079432992 x^{3}-79030988627868 x^{2} \\
& +25962912325800 x-3471675001929) \\
& L^{(3)}(y)=0 \text { is of order } 10 \text {. }
\end{aligned}
$$

Example3. Let's take the equation (example 4.9 in Ulmer 2003):

$$
\begin{gathered}
L(y)=\frac{d^{3} y}{d x^{3}}+\frac{7 x-3}{x(x-1)} \frac{d^{2} y}{d x^{2}}+\frac{2\left(138 x^{2}-115 x+7\right)}{27 x^{2}(x-1)^{2}} \frac{d y}{d x} \\
+\frac{10\left(162 x^{2}-28 x-7\right)}{729 x^{2}(x-1)^{2}} y
\end{gathered}
$$

It becomes as follow after using the transformation transfereL with Maple program (see transfereL Okko 2018):

$$
L_{S L}(y)=\frac{d^{3} y}{d x^{3}}+\frac{2}{27} \frac{12 x^{2}-7 x+7}{x^{2}(x-1)^{2}} \frac{d y}{d x}-\frac{2}{729} \frac{\left(324 x^{3}-301 x^{2}+525 x-224\right)}{x^{3}(x-1)^{3}} y
$$

whose third symmetric power $L^{(S)}(y)=0$ is of order 10 , and its differential Galois group $\mathfrak{S}(L)=G_{81}($ example 4.5 in Ulmer 2003 ). If $\left\{Y_{1}, Y_{2}, Y_{3}\right\}$ is a basis of the solution space of $L(y)=0$, then the invariants subspace of degree 3 of $G_{81}$ is generated by the polynomial $I_{1}=Y_{1} Y_{2} Y_{3}$ and the 3 semi-invariants spaces are generated by the polynomials $S_{1}=Y_{1}^{3}+Y_{2}^{3}+Y_{3}^{3}, S_{2}=Y_{3}^{3}+w^{3} Y_{1}^{3}+\left(-w^{3}-1\right) Y_{2}^{3}$, and $S_{3}=Y_{3}^{3}+\left(-w^{3}-1\right) Y_{1}^{3}+w^{3} Y_{2}^{3}$, (cf. Ulmer 2003 section 4.3), $w$ is the Wronskian. $s=x^{4}-2 x^{3}+x^{2}$ is an unique solution whose square is rational. By using our algorithm, we obtain the coefficients of the minimal polynomial $P(U)$ : 


$$
\begin{aligned}
& c_{3}=s=x^{4}-2 x^{3}+x^{2} \\
& c_{2}=s^{\prime}=4 x^{3}-6 x^{2}+2 x \\
& c_{1}=25313961574400 x^{11}-26409495661920 x^{10}+369414924755712 x^{9} \\
& -124939714582914 x^{8}+172153006123784 x^{7}-187209130557757 x^{6}+156749233965863 x^{5} \\
& -100541914181821 x^{4}+47033417633853 x^{3}-13872317475296 x^{2}+1989962456888 x \\
& -63264083456) / 54\left(11218295232 x^{9}-31314188304 x^{8}+84221681796 x^{7}-135184221739 x^{6}\right. \\
& +181586038333 x^{5}-181243862121 x^{4}+137006478371 x^{3}-78056142304 x^{2}+26777872800 x \\
& -3793656832) \\
& c_{0}=\left(73768485297024 x^{12}-333419069906784 x^{11}+855218148700536 x^{10}-369414924755712 x^{9}\right. \\
& +2139932814242671 x^{8}-2227665744927448 x^{7}+1741090046828426 x^{6}-963045565711138 x^{5} \\
& +324050153614243 x^{4}-1905494677324 x^{3}-56321850003132 x^{2}+22301556579456 x \\
& -2762481114400) / 729 x\left(34624368 x^{6}-64482264 x^{5}-143934343 x^{4}-155095486 x^{3}\right. \\
& \left.+138557839 x^{2}-79850400 x+16935968\right)\left(324 x^{3}-301 x^{2}+525 x-224\right)
\end{aligned}
$$

3. In this section we find the procedures which we have spoken about in the Algorithm (in section 2.) proposed in this paper.

\section{Code Maple}

1.

\#NormalForm1

with(diffalg):

with(Groebner):

with(PolynomialIdeals):

NormalForm $1:=\operatorname{proc}(\mathrm{y} 1, \mathrm{y} 2, \mathrm{y} 3, \mathrm{a} 0, \mathrm{a} 1)$

local x,i,s,R,R1,b0,b1;

global y10,y20,y30,y11,y21,y31,y12,y22,y32,a00,a10,a01,

$\mathrm{a} 02, \mathrm{a} 03, \mathrm{a} 04, \mathrm{a} 05, \mathrm{a} 06$;

$\mathrm{s}:=\mathrm{y} 1(\mathrm{x}) * \mathrm{y} 2(\mathrm{x}) * \mathrm{y} 3(\mathrm{x})$;

$\mathrm{R}[0]:=\mathrm{s}$;

$\mathrm{R} 1[0]:=\operatorname{subs}([\mathrm{y} 1(\mathrm{x})=\mathrm{y} 10, \mathrm{y} 2(\mathrm{x})=\mathrm{y} 20, \mathrm{y} 3(\mathrm{x})=\mathrm{y} 30], \mathrm{R}[0])$;

$\mathrm{b} 0:=\mathrm{a} 0(\mathrm{x}) ; \mathrm{b} 1:=\mathrm{a} 1(\mathrm{x})$;

for $\mathrm{i}$ from 1 to 9 do

if $\mathrm{i}>=3$ then $\mathrm{R}[\mathrm{i}]:=\operatorname{diff}(\mathrm{R}[\mathrm{i}-1], \mathrm{x})$;

$\mathrm{R}[\mathrm{i}]:=\operatorname{subs}(\operatorname{diff}(\mathrm{y} 1(\mathrm{x}), \mathrm{x} \$ 3)=\mathrm{b} 1 * \operatorname{diff}(\mathrm{y} 1(\mathrm{x}), \mathrm{x})+$

$\mathrm{b} 0 * \mathrm{y} 1(\mathrm{x}), \operatorname{diff}(\mathrm{y} 2(\mathrm{x}), \mathrm{x} \$ 3)=\mathrm{b} 1 * \operatorname{diff}(\mathrm{y} 2(\mathrm{x}), \mathrm{x})+\mathrm{b} 0 *$

$y 2(x), \operatorname{diff}(y 3(x), x \$ 3)=b 1 * \operatorname{diff}(y 3(x), x)+b 0 * y 3(x)$,

$\mathrm{R}[\mathrm{i}])$;

else

$\mathrm{R}[\mathrm{i}]:=\operatorname{diff}(\mathrm{R}[\mathrm{i}-1], \mathrm{x}) ; \mathrm{R}[\mathrm{i}]:=\operatorname{subs}(\operatorname{diff}(\mathrm{b} 1, \mathrm{x})=2 * \mathrm{~b} 0, \mathrm{R}[\mathrm{i}])$;

fi;

$\mathrm{R} 1[\mathrm{i}]:=\operatorname{subs}([\mathrm{y} 1(\mathrm{x})=\mathrm{y} 10, \mathrm{y} 2(\mathrm{x})=\mathrm{y} 20, \mathrm{y} 3(\mathrm{x})=\mathrm{y} 30, \operatorname{diff}(\mathrm{y} 1(\mathrm{x}), \mathrm{x})=\mathrm{y} 11$,

$\operatorname{diff}(y 2(x), x)=y 21, \operatorname{diff}(y 3(x), x)=y 31, \operatorname{diff}(y 1(x), x \$ 2)=y 12, \operatorname{diff}(y 2(x), x \$ 2)=y 22, \operatorname{diff}(y 3(x), x \$ 2)=y 32, b 0=a 00, b 1=a 10, \operatorname{diff}$ $(\mathrm{b} 0, \mathrm{x})=\mathrm{a} 01, \operatorname{diff}(\mathrm{b} 0, \mathrm{x} \$ 2)=\mathrm{a} 02, \operatorname{diff}(\mathrm{b} 0, \mathrm{x} \$ 3)=\mathrm{a} 03, \operatorname{diff}(\mathrm{b} 0, \mathrm{x} \$$

4) $=\mathrm{a} 04, \operatorname{diff}(\mathrm{b} 0, \mathrm{x} \$ 5)=\mathrm{a} 05, \operatorname{diff}(\mathrm{b} 0, \mathrm{x} \$ 6)=\mathrm{a} 06], \mathrm{R}[\mathrm{i}])$;

od; 
$\operatorname{return}([\operatorname{seq}(\mathrm{R} 1[\mathrm{i}], \mathrm{i}=0 . .9)])$;

end:

2.

\#NormalForm2

NormalForm2:=proc(11,12,order)

local j,NF ;global x1,x2,x3,x4,x5,x6,x7,x8,x9,x10,x11,x12,x13,

$\mathrm{x} 14, \mathrm{x} 15, \mathrm{x} 16, \mathrm{x} 17, \mathrm{x} 18, \mathrm{x} 19$;

for $\mathrm{j}$ from 1 to nops(11) do

NF[j-1]:=NormalForm(11[j],12,T);

od;

$\operatorname{return}([\operatorname{seq}([j-1, N F[j-1]], j=1 . .10)])$;

end:

$\mathrm{T}:=\operatorname{lexdeg}([\mathrm{y} 10, \mathrm{y} 20, \mathrm{y} 30, \mathrm{y} 11, \mathrm{y} 21, \mathrm{y} 31, \mathrm{y} 12, \mathrm{y} 22, \mathrm{y} 32],[\mathrm{w} 000$,

w100,w200,w110,w111,w222,w210,w220,w211,w221]):

B:=[w000-y10*y20*y30,w100-(y11*y20*y30+y10*y21*y30+y10*y20*

y31),w110-(y11*y21*y30+y11*y20*y31+y10*y21*y31),w200-(y12*

y20*y30+y10*y22*y30+y10*y20*y32),w210-(y11*y22*y30+y12*y21*

y30+y $12 * \mathrm{y} 20 * \mathrm{y} 31+\mathrm{y} 11 * \mathrm{y} 20 * \mathrm{y} 32+\mathrm{y} 10 * \mathrm{y} 22 * \mathrm{y} 31+\mathrm{y} 10 * \mathrm{y} 21 * \mathrm{y} 32), \mathrm{w} 111-$

(y11*y21*y31),w220-(y12*y22*y30+y12*y20*y32+y10*y22*y32),w211

$-(\mathrm{y} 12 * \mathrm{y} 21 * \mathrm{y} 31+\mathrm{y} 11 * \mathrm{y} 22 * \mathrm{y} 31+\mathrm{y} 11 * \mathrm{y} 21 * \mathrm{y} 32), \mathrm{w} 221-(\mathrm{y} 12 * \mathrm{y} 22 * \mathrm{y} 31+\mathrm{y} 12 *$

y21*y32+y11*y22*y32),w222-(y12*y22*y32)]:

$\mathrm{N} 2:=$ NormalForm2(N1,B,T);

\section{Code Magma}

1.

$/ / \mathrm{s}, \mathrm{a} 0, \mathrm{a} 1$ and their derivatives

Q:=RationalField();

p<w000,w100,w200,w110,w210,w111,w220,w211,w221,w222,a00,

$\mathrm{a} 10, \mathrm{a} 01, \mathrm{a} 02, \mathrm{a} 03, \mathrm{a} 04, \mathrm{a} 05, \mathrm{a} 06, \mathrm{~s} 0, \mathrm{~s} 1, \mathrm{~s} 2, \mathrm{~s} 3, \mathrm{~s} 4, \mathrm{~s} 5, \mathrm{~s} 6, \mathrm{~s} 7, \mathrm{~s} 8$,

s9,c0,c1>:=PolynomialRing(Q,30,"elim",10);

$\mathrm{N} 2:=[\mathrm{w} 000, \mathrm{w} 100, \mathrm{w} 200+2 * \mathrm{w} 110,6 * \mathrm{w} 111+3 * \mathrm{w} 210+3 * a 00 * \mathrm{w} 000+$

$\mathrm{a} 10 * \mathrm{w} 100, \ldots]$;

$/ / \mathrm{N} 2$ is the result of le resultat NormalForm2 procedure realized by Maple

id:=ideal<p|s0-N2[1],s1-N2[2],s2-N2[3],s3-N2[4],s4-N2[5],s5-

N2[6],s6-N2[7],s7-N2[8],s8-N2[9],s9-N2[10],c0-w111,c1-w110>;

GR:=GroebnerBasis(id);

2 .

//eliminate $\mathrm{c} 0$

$\mathrm{p} 1<\mathrm{c} 0, \mathrm{c} 1, \mathrm{a} 00, \mathrm{a} 10, \mathrm{a} 01, \mathrm{a} 02, \mathrm{a} 03, \mathrm{a} 04, \mathrm{a} 05, \mathrm{a} 06, \mathrm{~s} 0, \mathrm{~s} 1, \mathrm{~s} 2, \mathrm{~s} 3$,

s4,s5,s6,s7,s8,s9>:=PolynomialRing(Q,20,"elim",1);

sd:=[la suite des 6 derniers elements de la base de

Groebner GR];

id:=ideal $<\mathrm{p} 1 \mid \mathrm{sd}>$; 
Gr:=GroebnerBasis(id);

\section{References}

Benson, D. J. (1993). Polynomial Invariants of Finite Groups. Cambridge University Press.

Cox, D. J. (1992). Little \& D. O'Shea. Ideals, Varieties and Algoithms: An Introduction to Computational Algebraic Geometry and Commutative Algebra. Singer Verlag, Berlin and New York.

Fakler, W. (1997). Algorithms for Solving Linear Ordinary Differential Equations. Universität Karlsruhe, Germany 1997 .

Fakler, W. (1997). On second order homogeneous linear differential equations with Liouvillian solutions. Theoretical Computer Sciences 1997.

Kolchin, E. R. (1948). Algebraic matrix Groups and the Picard-Vessiot Theory of Homogeneous Ordinary Differential Equations. Annals of Math, 49.

Magid, A. (1994). Lectures on Differential Galois Theory. University Lecture Series of the American Mathematical Society, 1994.

Okko, N. (2018). Reproduction of Fuchs Relation for the Group $S_{4}^{S L_{2}}$ by Using Groebner Bases, Journal of Mathematics Research, 10(4), August 2018.

Singer, M. F., \& Ulmer, F. (1993). Galois Groups of Second and Third Order Linear Differential Equations, J. of Sym.Comp, 9-36.

Singer, M. F., \& Ulmer, F. (1997). Linear Differential Equations and Products of Lineare Forms. J. Pure and Applied Alg. 117 \& 118, 549-563, 1997.

Singer, M. F., \& Ulmer, F. (1998). Liouvillian and Algebraic Solutions of Second and Third Order Linear Differential Equations. North Carolina State University, 4 March 1998.

Sturmfels, B. (1993). Algorithms in Invariant Theory. Springer-Verlag Wien New York.

Sturmfels, B. (1993). Algorithms in Invariant Theory. Springer-Verlag Wien New York.

Ulmer, F. (1994). Irreducible Linear Differential Equations of Prime Order. J. of Sym.Comp, 18, 385-401.

Ulmer, F. (2003 ). Liouvillian Solutions of Third Differential Equations. J. of Sym.Comp, 36, 855-889.

\section{Copyrights}

Copyright for this article is retained by the author(s), with first publication rights granted to the journal.

This is an open-access article distributed under the terms and conditions of the Creative Commons Attribution license (http://creativecommons.org/licenses/by/4.0/). 